\title{
Effect of Uniaxial Stress upon Remanent Magnetization: Stress Cycling and Domain State Dependence
}

\author{
Jacques Revol, Ron Day, and Michael Fuller \\ Department of Geological Sciences, University of California, \\ Santa Barbara, California 93106, U.S.A.
}

(Received November 5, 1977)

\begin{abstract}
Polycrystalline magnetite and rock samples have been subjected to uniaxial compression and stress cycling at room temperature. The changes in the components of remanent magnetization were recorded continuously as a function of stress, and the changes in direction and total intensity of magnetization were inferred. Different types of response were recognized, according to the type of magnetization the sample was carrying, i.e., high field or weak field remanence. The anomalous increase of weak field remanence previously reported, appears partly reversible under stress cycling. However, the changes in the lowest stress range are irreversible and reduce the zero stress magnetization from cycle to cycle. Reversible rotations of the magnetization vector of as much as $180^{\circ}$ were observed during each half cycle and were primarily due to changes in the sign of the component of magnetization parallel to compression. An andesite whose magnetic phases are single domain according to hysteresis criteria showed a much smaller effect regardless of the type of magnetization it carried. These results again draw attention to the variety of stress responses and the importance of three component observations in field attempts to detect seismomagnetic precursors.
\end{abstract}

\section{Introduction}

The possibility of a seismomagnetic precursor arises because of the inverse piezo-magnetic effect exhibited by the magnetic minerals in stressed rocks. When changes in the magnetization of rocks take place at depth in the earth due to changes in stress, the magnetic ffeld at the earth's surface will be perturbed. Total field measurements of local geomagnetic field perturbations to an accuracy better than $0.1 \gamma$ (e.g., Smith and Johnston, 1976) are now possible, which is less than the field anomalies predicted by various models (STACEY and JOHNSTON, 1972; TALWANI and Kovach, 1972; Bhattacharyya, 1976). Magnetic anomalies associated with earthquakes in tectonically active areas have been reported by numerous authors (Breiner, 1964; Johnston et al., 1975; Smith and Johnston, 1976), and although doubts remain concerning the demonstration of the seismomagnetic effect, some of these results appear well founded. An accurate estimate of the seismomagnetic effect in a particular area requires knowledge of the rocks at depth in the crust, of the stress build up prior to the earthquake and of the effect of stress upon the magnetization of magnetic phases present in the rocks. Our work is directed at obtaining a better understanding of this magnetic response of rocks to stress. 
The effects of stress upon magnetic susceptibility and remanent magnetization have been widely investigated on rocks for stresses within the elastic range (KERN, 1961; Nagata, 1970; Pozzi, 1972; Stacey and Johnston, 1972). It has been found that, in general, the susceptibility parallel to compression decreases while the transverse susceptibility tends to increase. However there is a variety of the response which is not explicitly considered by these studies, as shown by KEAN et al. (1976). Uniaxial stress tends to give a rotation of remanent magnetization away from the stress axis and a decrease in the total intensity of magnetization. However, the available data are inadequate to define such important aspects of the phenomenon as its again size or domain state dependence.

Until recently, very little work has been done involving stresses exceeding the elastic range. The major aim of our work is to document the magnetic behavior of rocks stressed throughout the entire range to failure. As a first step we have used small cylindrical samples, subjected to uniaxial compression at room temperature without confining pressure. For each sample, the stress was increased from zero until failure occurred. The effects of stress upon induced or remanent magnetization were monitored continuously. We recognize that this is an unealistic experiment in terms of simulation of the failure likely in the crust, but in terms of interpretation of magnetic effects it is an important preliminary to the main investigation.

The most important conclusion to be drawn from our earlier study (REvol et al., 1977 ) is that magnetic effects of uniaxial compression up to failure are profound and readily observed in polycrystalline and in some rock types. Throughout the range of stress, substantial changes in remanence and susceptibility were seen. In the lower stress range there is a change in stress sensitivity, which may be related to the onset of dilatancy. There were also more erratic effects in the approach to failure. The appearance of the latter allowed us to predict failure systematically in the laboratory for those samples that showed any magnetic response to stress.

The application of stress caused changes in the components of both remanent magnetization and induced moment, with changes in the component parallel to compression dominating the response. Changes in the intensity and direction of the magnetization vector are related to the initial orientation of magnetization, as well as to the type of magnetization the sample is carrying. In the case of strong field remanent magnetization $\left(\mathrm{IRM}_{s}\right)$ the total intensity of magnetization decreased monotonically with increasing stress in zero field. In contrast, demagnetized samples, and samples carrying a weak field thermo-remanent magnetization (TRM), showed an anomalous increase in total intensity with increasing stress in zero field, due principally to the increase parallel to compression. This effect produced large changes in the direction of the magnetic moment (Fig. 1). The magnitude of this increase, and the extent of the stress range over which it occurs appear to be related to be the magnitude and direction of magnetization before stressing. A serpentinite sample carrying natural remanent magnetization also showed this anomalous increase in intensity under stress in zero field.

The stress effect upon susceptibility was earlier shown to be strongly dependent 

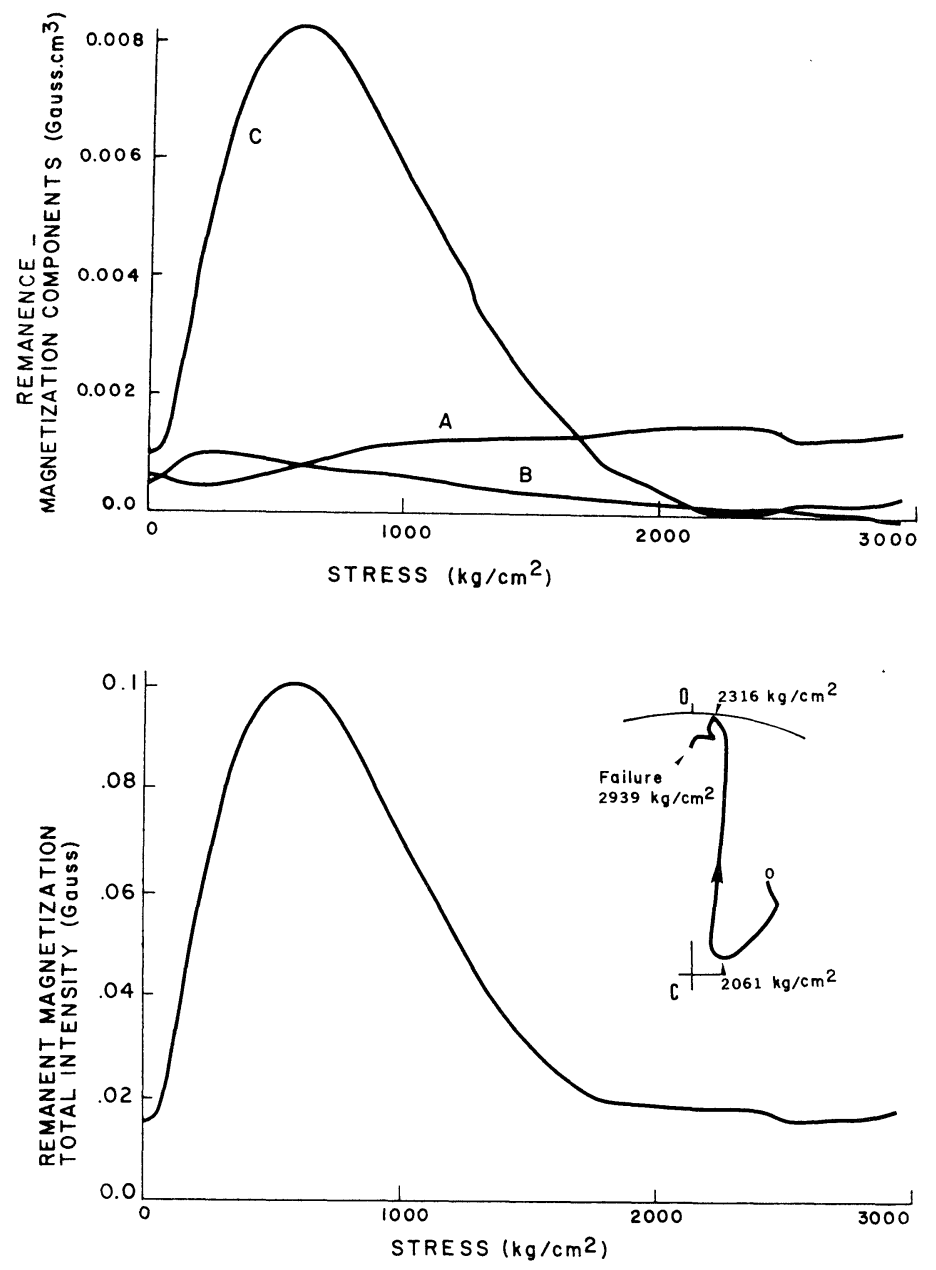

Fig. 1. Components, intensity and rotation of remanent magnetization under stress for multidomain magnetite carrying a TRM.

upon grain size (KEAN et al.,1976). Thus synthetic samples of coarse grain magnetite $(150-175 \mu \mathrm{m})$ gave stress sensitivities of $8 \times 10^{-4} \mathrm{~cm}^{2} \cdot \mathrm{kg}^{-1}$, while $1-2 \mu \mathrm{m}$ dispersions gave $0.3 \times 10^{-4}$. Synthetic titanomagnetites showed an even greater variation from $4 \times 10^{-3}$ to $0.3 \times 10^{-4} \mathrm{~cm}^{2} \cdot \mathrm{kg}^{-1}$. Rock samples showed less variability with values ranging from $6 \times 10^{-4}$ to $1 \times 10^{-4} \mathrm{~cm}^{2} \cdot \mathrm{kg}^{-1}$, with the coarse grain titanomagnetitebearing samples giving the larger value. These values are comparable with those cited by StaCeY and Johnston (1972).

The stress sensitivity for remanence was also given by STACEY and JoHnston (1972) and values of between $0.7 \times 10^{-4}$ and $18.7 \times 10^{-4} \mathrm{~cm}^{2} \cdot \mathrm{kg}^{-1}$ were cited with rocks with the more titaniferous magnetite being more sensitive. The discovery of the anomalous increase in remanence parallel to compression, in polycrystalline magnetite and rocks, carrying weak field remanence, complicates the description of 
the stress sensitivity of remanence considerably. If calculated from $M_{(\sigma)} / M_{0}=$ $1 /\left(1+S_{M} \sigma\right), S_{M}$ changes sign and magnitude according to the stress range, with a maximum value of $3 \times 10^{-3} \mathrm{~cm}^{2} \cdot \mathrm{kg}^{-1}$ between 0 and 300 bars. It appears that the slope at a particular stress, normalized by the magnetization at that stress, $\left(1 / M_{(\sigma)}\right)(\partial M / \partial \sigma)$, is a more meaningful form and is equivalent to that normally quoted for the low stress range. The values for this stress sensitivity parameter vary from $+3 \times 10^{-3} \mathrm{~cm}^{2} \cdot \mathrm{kg}^{-1}$ through zero to a comparable negative value (Fig. 1). Although not as large in rock samples, similar effects have been seen, e.g., in serpentinite.

The presence of the anomalous increase parallel to compression means that the stress sensitivity of remanence can be considerably larger than that of susceptibility. For example, in the polycrystalline magnetite the maximum susceptibility sensitivity of $4 \times 10^{-4}$ is almost an order less than the maximum remanence sensitivity. Before we can translate this into an assessment of the relative importance of the seismomagnetic effect, the roles of susceptibility and remanence in determining a magnetic field anomaly must be defined by the Koenigsberger ratio

$$
Q=\frac{\mathrm{NRM}}{k H_{\text {earth }}} \approx \frac{\mathrm{NRM}}{k 0.5} .
$$

Thus, for the polycrystalline magnetite with a typical value of TRM of 0.05 Gauss and a susceptibility of 0.15 Gauss/Oe, the remanent effect will dominate over the stress ranges for which the anomalous increase grows and decays. In the case of the serpentinite the remanence effect will also dominate.

In this paper, we describe additional experiments primarily related to cycling, to determine if the anomalous increase in total intensity of remanent magnetization (usually due to an increase in the component parallel to compression) is reversible. We also distinguish between fine-particle and multidomain behavior under stress.

As in our earlier experiments we have not made simultaneous measurements of strain and components of magnetization, nor have we introduced a confining pressure.

\section{Experimental Procedure}

Our experimental technique allows for the continuous measurement of the changes in the three components of magnetization vs. applied uniaxial stress. The equipment and procedures have been described earlier (Revol et al., 1977). Modifications have been introduced to speed up the process of data reduction.

The output signals from the magnetometer and pressure transducer are now sent to an analog to digital converter, the output of which is connected to a teletype. A reading of the four parameters can be made approximately every half second, but averages can be taken over a variable number of readings to give quasi-continuous recording.

The data are then reduced to obtain the total intensity of magnetization vs. stress, and the directional changes of the vector are plotted on a stereonet. The axis 
of compression ( $C$ axis) is perpendicular to the plane of the projection of the net. The initial azimuthal direction is arbitrary and only the relative changes in direction are of interest.

\section{Magnetic Characteristics of Samples}

The rock samples used in these experiments were in the form of small cylinders with a length to diameter ratio of about 1.29 , a value around which the most uniform stress distribution is obtained. This also gives a shape anisotropy that should not exceed $10 \%$.

The following rock types were used:

1) Natural polycrystalline magnetite (Norway).

Diameter $=0.43 \mathrm{~cm}$, Length $=0.57 \mathrm{~cm}$.

Saturation magnetization $J_{s}=95.0 \mathrm{emu} / \mathrm{g}$,

Remanent magnetization $J_{r}=1.1 \mathrm{emi} / \mathrm{g}$,

$J_{r} / J_{s}=0.01$, coercive force $H_{c}=17 \mathrm{Oe}$,

Remanent coercive force $H_{r c}=145 \mathrm{Oe}, H_{r c} / H_{c}=8.5$.

The ratios $H_{r c} / H_{c}$ and $J_{r} / J_{s}$ indicate multidomain behavior for this material, also suggested by the shape of the A.F. demagnetization curve.

2) Porphyritic andesite from the margin of a dike.

Wildwood Park, California.

Diameter $=0.43 \mathrm{~cm}$, Length $=0.56 \mathrm{~cm}$.

$J_{s}=2.77 \mathrm{emu} / \mathrm{g}$ of sample, $J_{r}=1.72 \mathrm{emu} / \mathrm{g}$ of sample,

$J_{r} / J_{s}=0.62, H_{c}=323 \mathrm{Oe}, H_{r c}=400 \mathrm{Oe}, H_{r c} / H_{c}=1.24$.

These values indicate that this rock is mainly composed of single domain magnetic carriers. The Curie point is $340 \pm 10^{\circ} \mathrm{C}$ indicating a $0.4 x$ value titanomagnetite. The opaques are about $2 \mu \mathrm{m}$ or less in diameter.

\section{Results}

\subsection{Stress cycling}

In this section we describe the series of experiments designed to determine if the previously reported anomalous increase in magnetization (REvol et al., 1977) is reversible for different remanent magnetizations.

\subsubsection{Magnetite carrying isothermal remanent magnetization (IRM)}

The application of uniaxial stress in zero field produced a decrease in each of the three components of magnetization. The total intensity of magnetization was reduced by an order of magnitude during the first cycle. The rate of decrease was high at low stresses but decreased progressively in the range corresponding to half the breaking stress. This type of behavior has already been observed in the elastic range (NAGATA, 1969), but evidently it also applies when the sample is taken near to failure. The behavior of the three components, intensity and rotation of the 

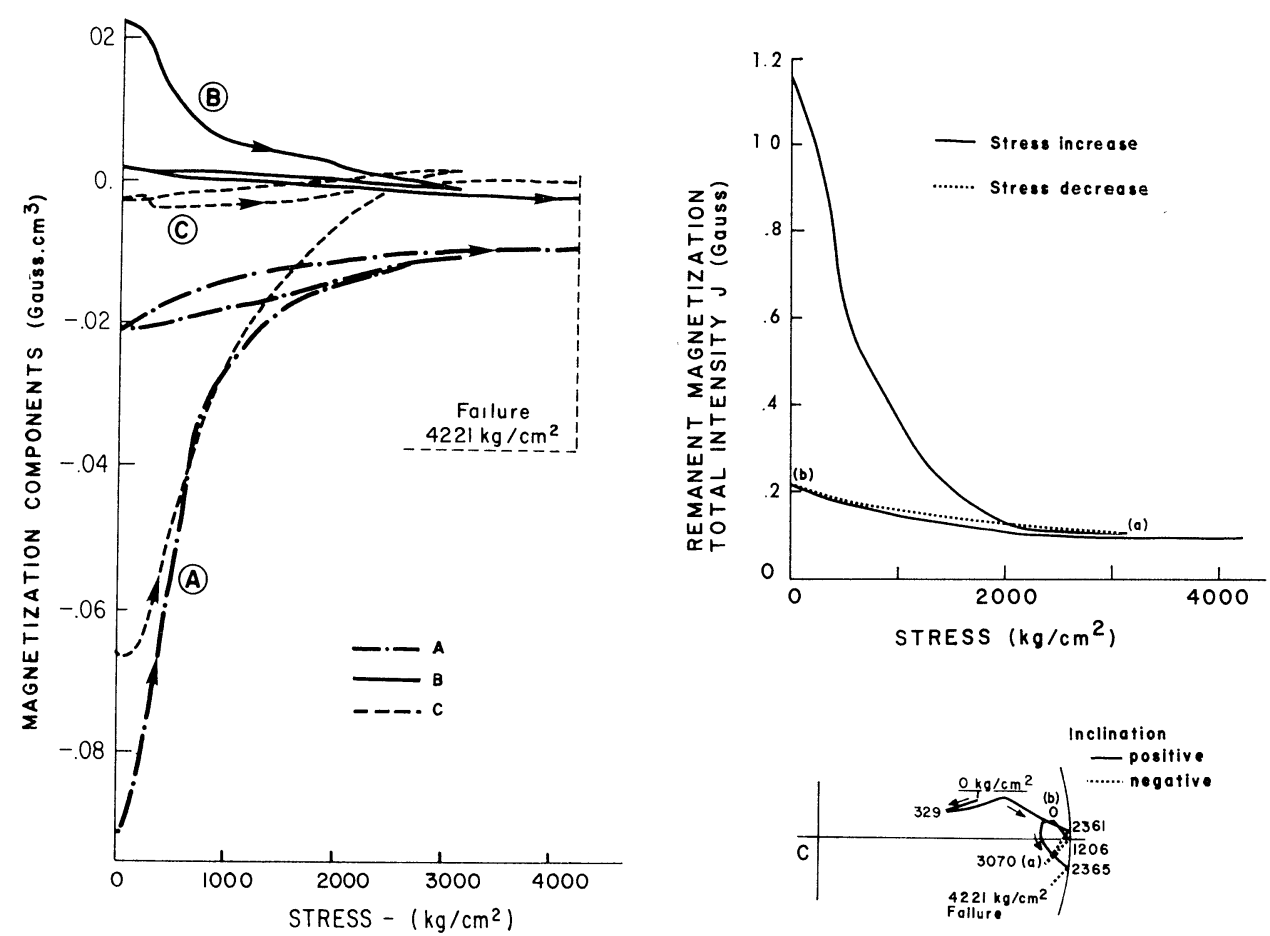

Fig. 2. Changes in the components of remanent magnetization due to stress cycling between 0 and $1,200 \mathrm{~kg} / \mathrm{cm}^{2}$ for multidomain magnetite carrying an IRM.

magnetization vector is shown in Fig. 2. Stressing produced changes of about $45^{\circ}$ in both declination and inclination.

It is worth mentioning that in this case the sample was cycled to a high stress value, resulting in an increase of the ultimate failure stress. This can be expected, and is probably due to cold working of the sample.

\subsubsection{Magnetite carrying thermo-remanent magnetization (TRM)-Intermediate stress range cycling}

A sample was given a TRM by heating to $600^{\circ} \mathrm{C}$ in a vacuum and cooling in a 0.4-Oe field.

This sample was cycled 5 times from 0 to approximately $1,200 \mathrm{~kg} / \mathrm{cm}^{2}$ and then stressed to failure. The changes in the three components of magnetization brought about by the applied stress are shown in Fig. 3a. The plot of total intensity vs. stress and the directional changes of the magnetization vector are given in Fig. $3 \mathrm{~b}$. The numbers on the curves in Figs. $3 \mathrm{a}$ and $3 \mathrm{~b}$ indicate the successive stress cycles. The irreversible effect of stress on the $A$ component, which was perpendicular to compression, was to reduce its value at zero stress from cycle to cycle. During a particular stress cycle, the $A$ component decreased in value with the application of stress, and passed through zero without any apparent change in behavior.

Cycling produced an essentially reversible increase in the $B$ component, which 
was also perpendicular to compression, but a decrease was observed before the sample reached failure.

Under stress, the $C$ component, which was parallel to compression, decreased in absolute value, passed through zero magnetization and continued to increase in the opposite sense with further pressure. After each cycle, the magnetization at zero stress was progressively reduced in absolute value. It eventually changed sign after the fourth cycle. During the fifth cycle the pressure was released too fast to permit accurate determination of the magnetization between 1,200 and $0 \mathrm{~kg} / \mathrm{cm}^{2}$. The large offset observed between points 9 and 10 of Fig. 3a could therefore be an artifact but could also be explained by cracking.

Figure $3 \mathrm{~b}$ reveals a decrease in total intensity of about $75 \%$ of the initial value during the first cycle, followed by an increase to approximately the original value. The minimum was observed at a stress corresponding to one-third of the breaking stress. Further cycling reduced progressively the zero stress magnetization by about $90 \%$ of the initial value, but the subsequent increase was predominantly reversible. The questionable offset between points 9 and 10 prevents any definite conclusion about the last stress cycle; however it appears that the magnetization reached a maximum greater than the initial intensity, when the sample was stressed to failure.

Directional changes were largely reversible for at least the first three cycles, with little azimuthal deviation. The magnetization vector rotated away from the compression axis, and passed through the plane perpendicular to compression, which is the plane of projection of the stereonet (Fig. 3b). It then moved back towards the axis of compression, to a direction opposed to the initial vector for a stress of about $1,200 \mathrm{~kg} / \mathrm{cm}^{2}$. When the stress was released the path was retraced. During stress release on the fourth cycle the vector rotated back by only about $80^{\circ}$, and followed the same trajectory in reverse when the stress was increased again.

\subsubsection{Magnetite carrying TRM-Low stress range cycle}

This sample was given TRM under the same conditions as described before. It was cycled between 0 and $400 \mathrm{~kg} / \mathrm{cm}^{2}$, and the changes in total intensity and the directional changes are given in Fig. 4.

The first three cycles reduced the zero stress magnetization. The corresponding directional changes were reversible. The stress was then increased to about $900 \mathrm{~kg} / \mathrm{cm}^{2}$, which resulted in rotation of the magnetization vector away from the compression axis and switched the sign of the inclination. The release of stress gave a zero stress magnetization less than half the initial value (points 3 and 4). Three more cycles were carried out between 0 and $400 \mathrm{~kg} / \mathrm{cm}^{2}$ (points 4 and 5). The changes in intensity as well as the directional changes were reversible during these cycles, with the magnetization vector alternating between a positive and a negative inclination.

The last stress increase produced a large increase in magnetization to give a value almost identical to the initial value at a stress of about one-third of the breaking stress. A comparable decrease in intensity was observed before the sample reached failure. The corresponding directional change involved rotation of the 

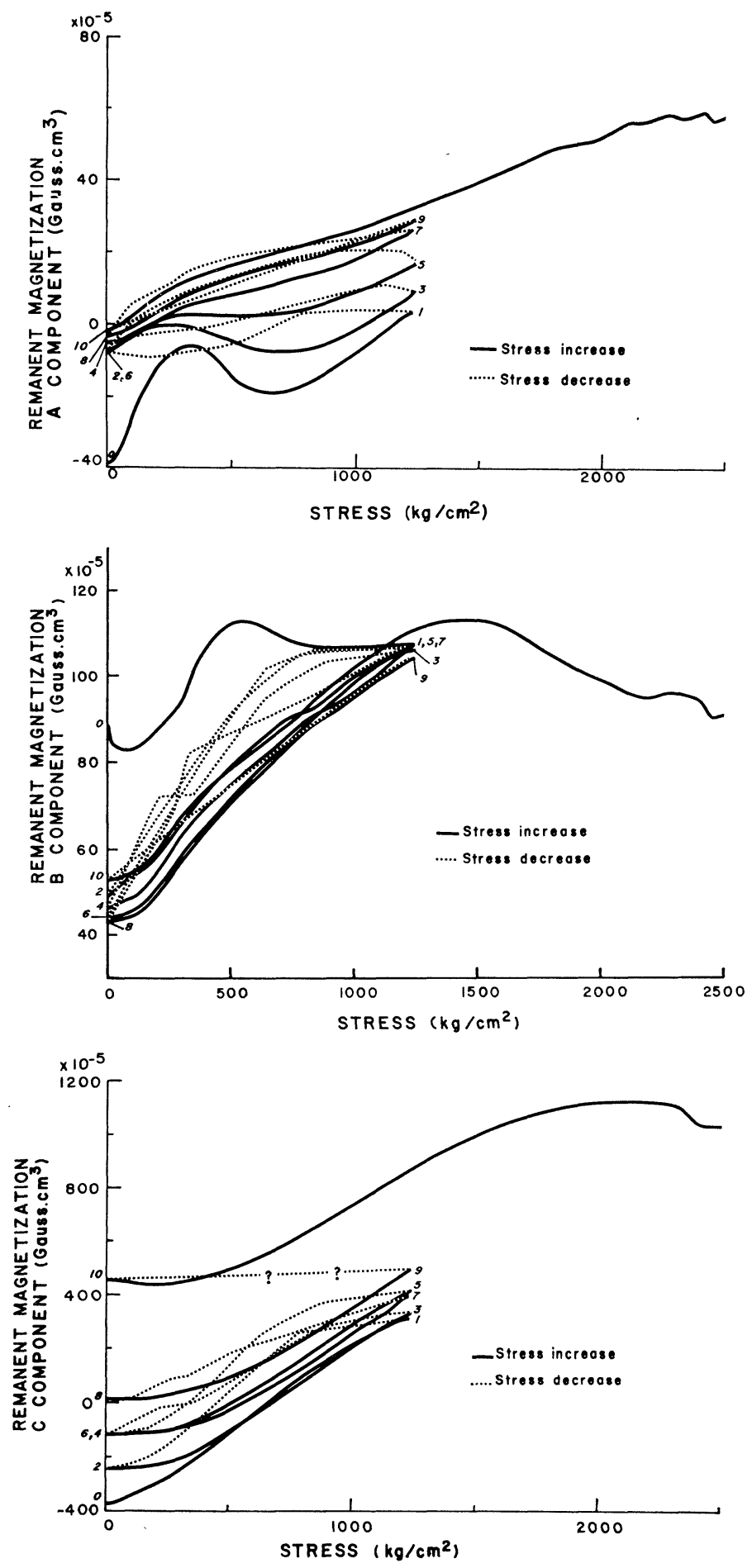

Fig. 3a 

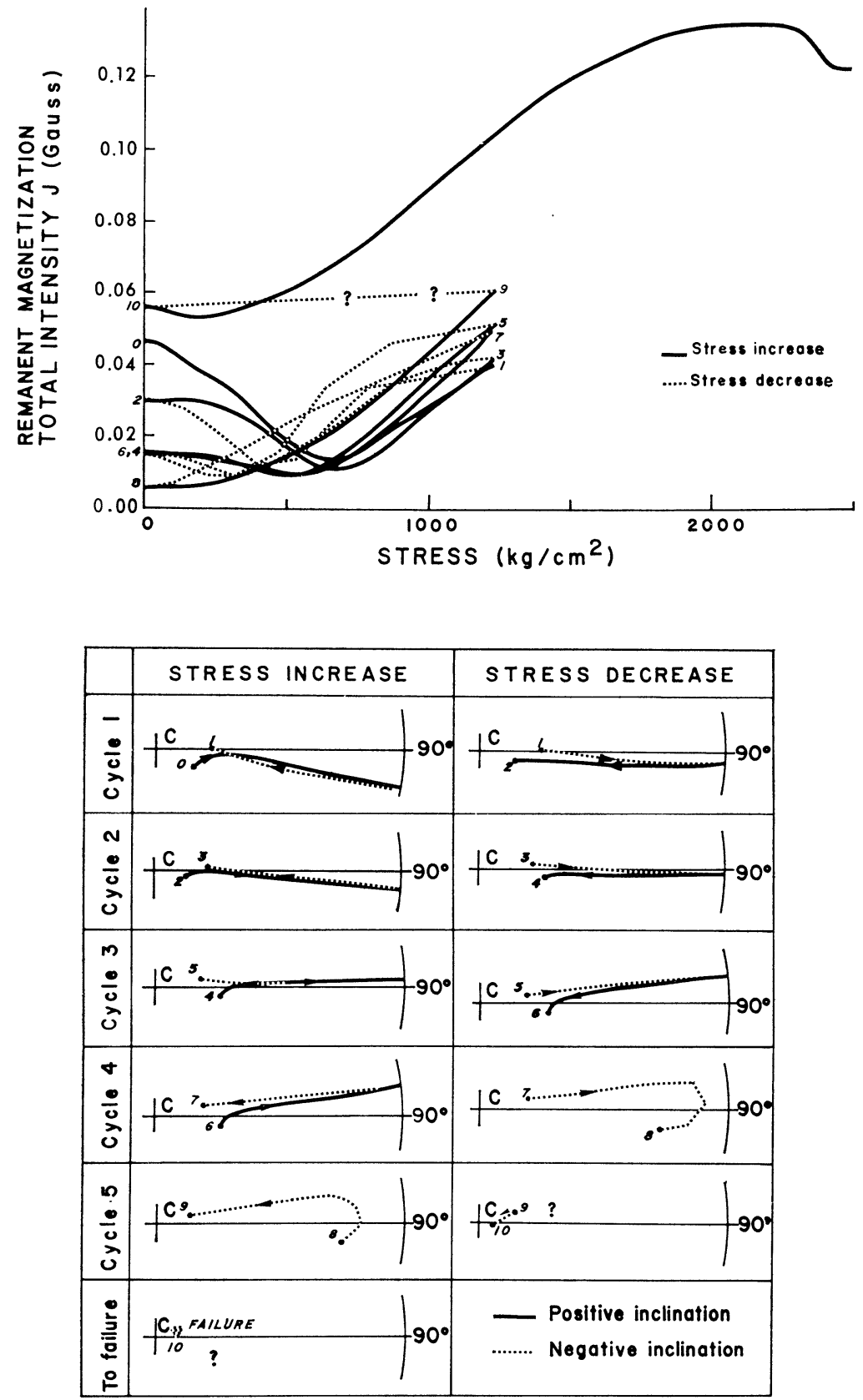

Fig. 3b

Fig. 3a. Changes in the components of remanent magnetization due to stress cycling between 0 and $1,200 \mathrm{~kg} / \mathrm{cm}^{2}$ for magnetite carrying a TRM.

Fig. 3b. Changes in total intensity of remanent magnetization and directional changes due to stress cycling between 0 and $1,200 \mathrm{~kg} / \mathrm{cm}^{2}$ for magnetite carrying a TRM. 

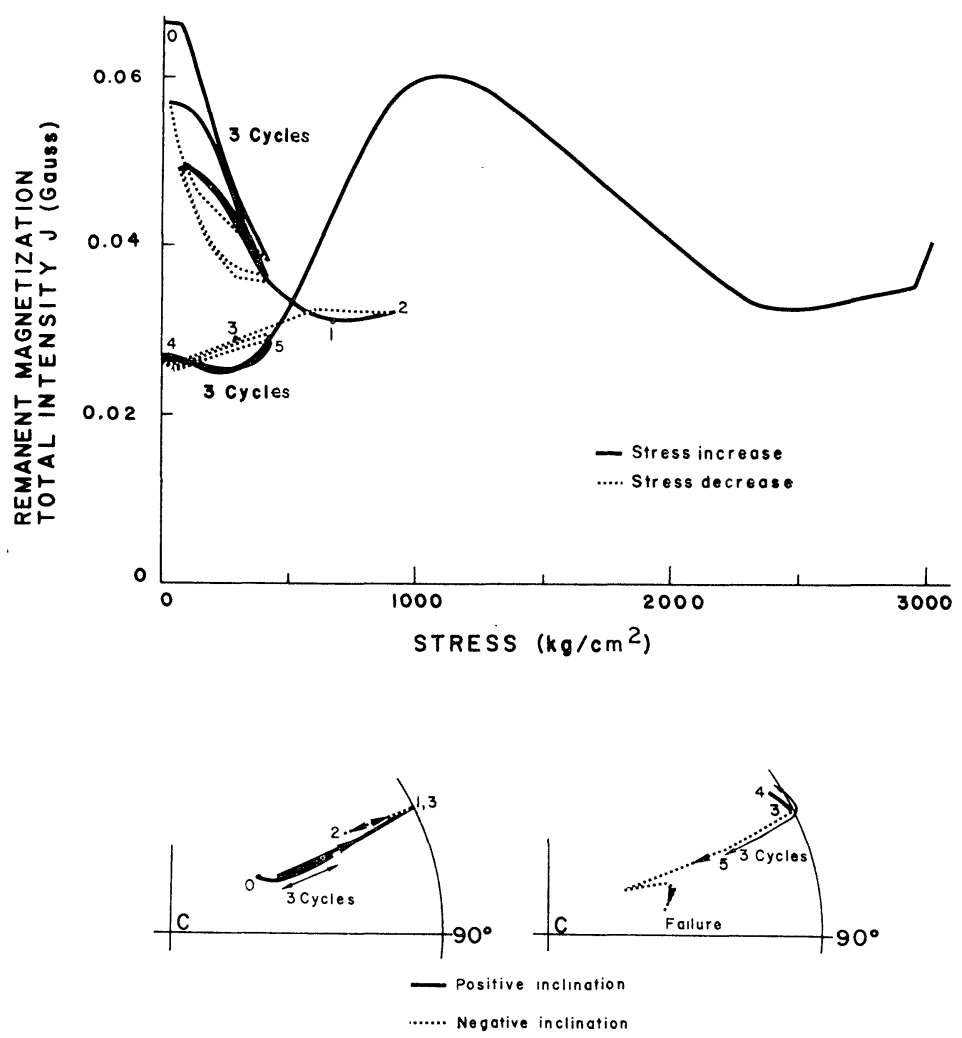

Fig. 4. Changes in total intensity of remanent magnetization and directional changes due to stress cycling between 0 and $400 \mathrm{~kg} / \mathrm{cm}^{2}$ for magnetite carrying a TRM.

magnetization vector toward the axis of compression in an opposed sense to that of the initial orientation.

\subsubsection{Magnetite carrying TRM-Cycling between 1,000 and $1,200 \mathrm{~kg} / \mathrm{cm}^{2}$}

This sample carried a TRM similar to those previously described, and was cycled in an intermediate stress range. For stresses lower than the cycling range, all three components of magnetization initially decreased in absolute value. For stresses exceeding the cycling range, the component parallel to compression and one of the components perpendicular to it, increased in absolute value, whereas the second component perpendicular to compression kept decreasing in absolute value. None of the components actually crossed the zero magnetization value, contrary to cases described above.

The intensity of magnetization decreased by $60 \%$ of the initial value and started to increase for stresses just below the stress cycling range. Cycling five times gave approximately reversible changes in magnetization, producing only a slight decrease from cycle to cycle. For stresses exceeding the cycling range in the final approach to failure, the intensity increased again before the appearance of an erratic behavior 
announcing failure. The magnetization rotated first away from the compression axis, and then back toward it at stresses higher than the cycling interval.

This sample did not show, in the lower stress range, the large increase observed in other samples carrying the same type of magnetization (TRM). However a smaller increase in magnetization was observed at higher stresses. It may actually be the same effect, shifted with respect to stress by inhomogeneity of internal stress.

\subsection{Grain size control of response to uniaxial stress}

In our earlier paper (REVol et al., 1977) we reported that a granodiorite sample containing fine magnetic carriers with saturation IRM showed virtually no change

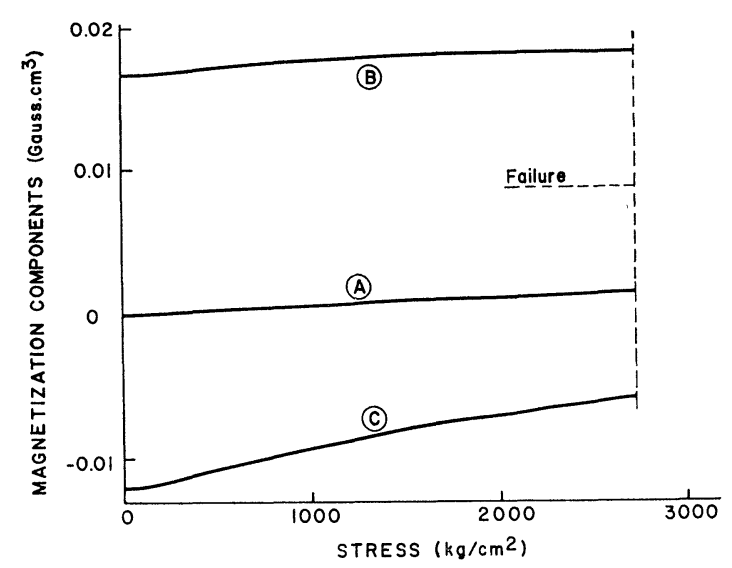

Fig. 5. Changes in the components of remanent magnetization under stress for andesite containing single domain particles and carrying a saturation IRM.

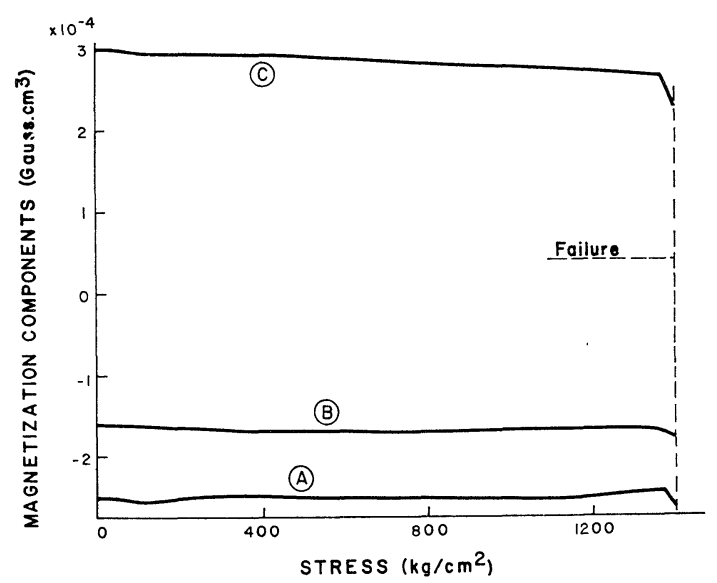

Fig. 6. Changes in the components of remanent magnetization under stress for andesite containing single domain material and carrying a TRM. 
under stress. Stress cycling was also studied on this sample and showed no significant effect. Additional experiments discussed below have been conducted with samples containing fine magnetic carriers.

The andesite described above and containing fine titanomagnetite particles carrying a saturation IRM showed only a small effect due to the applied stress (Fig. 5). The components of magnetization perpendicular to compression increased slightly in absolute value while the component parallel to compression decreased. The total intensity of magnetization decreased very little and the magnetization vector slightly rotated away from the compression axis.

The andesite was given a TRM under the same conditions as magnetite. The plot of the components of magnetization vs. stress is given in Fig. 6. The component parallel to compression decreased under stress but no noticeable change was observed in the components perpendicular to compression. The total intensity of magnetization showed a small decrease at the approach to failure, and no change in direction was observed.

\section{Conclusions}

The present paper establishes the degree of reversibility of the previously reported anomalous increase in TRM of polycrystalline magnetite under stress cycling, in zero field and at room temperature. It is clear that whereas the changes in the lowest stress range are irreversible and tend to reduce the magnitude of the zero stress magnetization after cycling (also reported by HeNYeY et al., 1978), the anomalous increase which occurs in a somewhat higher stress range is reversible. The importance of this result is that the effect may be expected to persist throughout many natural cycles. In multidomain magnetite this anomalous response of remanent magnetization will be greater than the changes in susceptibility.

The distinction between the response of multidomain and fine grain single domain magnetic material is remarkable. The latter shows very small responses in both susceptibility and remanent magnetization. For example, as we noted in the introduction, the earlier work of KEAN et al. (1976) suggests values of order $10^{-5} \mathrm{~cm}^{2} \cdot \mathrm{kg}^{-1}$ for the stress sensitivity for susceptibility. The results from this paper suggest that if a similar sensitivity parameter is used, the values for TRM carried by single domain material will also be of order $10^{-5} *^{*}$ In contrast, the multidomain remanence effect is initially two orders larger in the polycrystalline magnetite and appears long before failure. Moreover, the form of the effect does not actually permit useful definition of the stress sensitivity in the manner used previously. Both in the polycrystalline magnetite and in rock samples containing multidomain magnetite the remanent moment effect dominates over the susceptibility effect in the earth's field. In fact, in comparing the stress effect in the presence and absence of a field we have found little difference. Thus, in the polycrystalline magnetite we have

* This very small stress effect upon weak field remanence of single domain material suggests that only in extreme circumstances will the stable NRM of rocks be affected by stress. 
studied, the magnetic response to a stress of kilobars unconfined uniaxial compression is dominated by the soft multidomain material. If such material carries a weak field NRM or TRM it is the anomalous increase parallel to compression which is the principal effect. It now remains to find out how general this phenomenon is, particularly if it is seen when the sample is confined, and if so, whether it can eventually be used as a seismomagnetic precursor.

This work was supported by a grant from the U.S. Geological Survey. The authors are grateful to $V$. Sefcik for interfacing the equipment with a mini-computer.

\section{REFERENCES}

Bhattacharyya, B.K., Development of an Optimized Experiment by Measuring Piezomagnetic Signals of Geological Origin, Space Sciences Laboratory Series 16, Issue 27, June 2, 1976.

Breiner, S., Piezomagnetic effect at the time of local earthquakes, Nature, 202, 790-791, 1964.

HenYey, T.L., S.J. PIKe, and D.F. PALMER, On the measurement of stress sensitivity of NRM using a cryogenic magnetometer, J. Geomag. Geoelectr., 30, 607-617, 1978.

Johnston, M.J.S., G.D. Myren, N.W. O'HARE, and J.H. Rodgers, A possible seismomagnetic observation on the Garlock fault, California, Seismol. Soc. Am. Bull., 65, 1129-1132, 1975.

KeAn, W.F., R. Day, M. Fuller, and V.A. Schmidt, The effect of uniaxial compression on the initial susceptibility of rocks as a function of grain size and composition of their constituent titanomagnetites, J. Geophys. Res., 81, 861-872, 1976.

KERN, J.W., The effect of stress on the susceptibility and magnetization of a partially magnetized multidomain system, J. Geophys. Res., 66, 3807-3816, 1961.

NAGATA, T., Basic magnetic properties of rocks under the effects of mechanical stresses, Tectonophysics, 9, 167-195, 1970.

Pozzr, J.P., Piezomagnetic properties of hematite and effect of reheating, Ann. de Geophysique, 28, 1-13, 1972 (in French).

Revol, J., R. DAy, and M. Fuller, Magnetic behavior of magnetite and rocks stressed to failureRelation to earthquake prediction, Earth Planet. Sci. Lett., 37, 296-306, 1977.

SMITH, B.E. and M.J.S. JohNSTON, A tectonomagnetic effect observed before a 5.2 earthquake near Hollister, California, J. Geophys. Res., 81, 3556-3560, 1976.

STACEY, F.D. and M.J.S. JOHnSTON, Theory of the piezomagnetic effect of titanomagnetite-bearing rocks, Pure Appl. Geophys., 97, 146-155, 1972.

Talwani, P. and R.L. Kovach, Geomagnetic observations and fault creep in California, Tectonophysics, 14, 245-256, 1972. 